\title{
Factors affecting radiographic progression of knee osteoarthritis
}

\author{
Joanna Ledingham, Marian Regan, Adrian Jones, Michael Doherty
}

\begin{abstract}
Objectives-To evaluate the prognostic significance of patient characteristics and radiographic features at the knee for outcome of knee osteoarthritis.

Methods-This was a prospective observational study of 350 osteoarthritic knees. Clinical and radiographic data were obtained on 188 hospital referred patients (mean age 70, range 34-91 years). Results-Median duration of follow up was two years (range 1-5 years). The majority of patients $(48 \%)$ reported deterioration, but $23 \%$ experienced improvement in symptoms during the study period. Reported exercise tolerance remained unchanged in the majority $(62 \%)$ and deteriorated in $35 \%$. Change in at least one individual radiographic feature of osteoarthritis was seen in $\mathbf{2 5 2}$ $(72 \%)$ knees: increase in joint space narrowing occurred in $52 \%$, osteophyte in $32 \%$, cysts in $19 \%$, sclerosis in $14 \%$, and attrition in $30 \%$. Increase in Kellgren grade occurred in 137 (39\%) knees. Knee effusion, osteoarthritis at multiple joint sites, and nodal change associated with change in Kellgren grade (odds ratios 1.03, $2 \cdot 39$, and $1 \cdot 80 ; 95 \%$ confidence intervals (CI) 1.01 to $1.05,1.16$ to 4.93 , and 1.02 to $3 \cdot 17$, respectively); warmth at the knee associated with change in any radiographic feature (odds ratio $2 \cdot 22 ; 95 \%$ CI $1 \cdot 19$ to $4 \cdot 14)$. Development of, or increase in, attrition and joint space narrowing associated with worsening symptoms and function and occurred with increased frequency in knees with effusions, clinical warmth and calcium pyrophosphate crystals in synovial fluid $(p<0.05)$.

Conclusions-A high rate of change, radiographic more than clinical, was seen in osteoarthritic knees during this study. Poor clinical and radiographic outcome associated with calcium pyrophosphate crystal deposition and clinical inflammation as reflected by knee effusion and warmth.
\end{abstract}

(Ann Rheum Dis 1995; 54: 53-58)

The heterogeneity of osteoarthritis is increasingly recognised and a number of "subsets" have been proposed according to presumed initiating factors (for example trauma), the number and pattern of joints involved (for example nodal generalised osteoarthritis $^{1}$ ), distribution within jonts, ${ }^{2-7}$ differing radiographic appearances and the presence of associated calcium crystal deposition. ${ }^{7-12}$ At the knee a number of factors such as obesity or meniscectomy ${ }^{13}{ }^{14}$ are now known to predispose to development of osteoarthritis, but factors that associate with its progression are less clear. Intuitively, it seems likely that similar factors associate with initiation and with progression, but this is not necessarily the case.

Few studies have documented the natural history of knee osteoarthritis ${ }^{4{ }^{15-17}}$ and factors that may associate with progression at this site remain speculative. This study in patients referred to hospital because of knee osteoarthritis was undertaken to evaluate the prognostic significance of radiographic features at the knee, in addition to other local and general factors that have been used to subdivide osteoarthritis.

\section{Patients and methods}

This study was approved by the local research ethics committee. Consecutive patients attending one general rheumatology clinic with symptomatic knee osteoarthritis were initially recruited into a cross-sectional study of knee osteoarthritis. ${ }^{18}$ Seventy five percent of these patients underwent prospective review for at least one year.

\section{DIAGNOSTIC CRITERIA}

Knee osteoarthritis was radiographically defined as presence of joint space narrowing with osteophyte in one or more compartment (patellofemoral, medial tibiofemoral, lateral tibiofemoral). Alternative arthropathies were excluded by clinical examination, blood tests, synovial fluid microscopy, and radiographic screening. ${ }^{18}$

INITIAL ASSESSMENTS

Historical-Each patient underwent a full rheumatological history and examination with particular emphasis on the presence of activity, rest and nocturnal knee pain (each graded $0-3$ for each knee: $0=$ none, $1=$ mild, $2=$ moderate $3=$ severe), duration of symptoms and past history of previous major trauma, meniscectomy, or other surgery to the knees.

Clinical-Knees and all non-axial joints were examined for clinical abnormality consistent with osteoarthritis. At the knee individual characteristics including effusion, increased warmth, and instability were recorded.$^{18}$ Nodal change was defined clinically as presence of 
typical superolateral Heberden's or Bouchard's nodes, or both, affecting at least three rays of each hand.

Synovial fluid-Attempted aspiration of synovial fluid was undertaken from all affected knees regardless of whether effusions were clinically apparent. Calcium pyrophosphate crystals were identified by compensated polarising light microscopy.

Radiographic-In each patient standardised plain radiographs were taken of the knees: standing anteroposterior radiograph of the tibiofemoral joint $(55 \mathrm{kV}, 8 \mathrm{~mA} \mathrm{~s}$, full scale deflection (FSD) $100 \mathrm{~cm}$ ) plus lateral views in $30^{\circ}$ flexion of the patellofemoral joint $(55 \mathrm{kV}$, $8 \mathrm{~mA} \mathrm{~s}$, FSD $100 \mathrm{~cm}$ ). In addition, radiographs were taken of the pelvis (supine anteroposterior), hands (to include wrists), shoulders, lumbar spine (anteroposterior and lateral), and thoracic spine (anteroposterior), together with any other joint involved clinically.

Initial films were examined and scored by two observers ( $J \mathrm{~L}, \mathrm{MR}$ ) as previously described. ${ }^{18}$ Radiographic severity of osteoarthritis at each knee compartment and at other joints was graded according to the Kellgren and Lawrence system. ${ }^{19}$ For any joint, osteoarthritis was defined by the presence of joint space narrowing (equivalent to Kellgren grade 2); severe osteoarthritis was defined as obliteration of joint space (equivalent of Kellgren grade 4). Polyarticular interphalangeal osteoarthritis was defined by radiographic involvement of interphalangeal joints in at least three rays of both hands. Chondrocalcinosis was recorded if present. The bone response at each knee was categorised as either atrophic or hypertrophic; ${ }^{20}$ the category 'indeterminate' was used for knees demonstrating a mixed pattern. In addition, individual features of osteoarthritis were graded $0-3$ using a modification of Thomas's $\operatorname{method}^{7}$ for each knee compartment.

REVIEW ASSESSMENTS

Patients were clinically reassessed at six month intervals. Patients gave a global assessment of change in symptoms (better, same, worse), and pain scores at completion of the study were compared with initial scores. Functional status was assessed at each visit using the Stein-

Table 1 Characteristics of the 188 study patients

\begin{tabular}{lcl}
\hline Characteristic & No. & \% or range \\
\hline Gender: Male & 70 & 37 \\
$\quad$ Female & 118 & 63 \\
Median age (yr) & 71 & $34-91$ \\
Median symptom duration (yr) & 9 & $1-52$ \\
Body mass index & $27 \cdot 8$ & $17 \cdot 5-42 \cdot 4$ \\
Nodal change & 46 & 24 \\
Knee OA & & \\
Unilateral & 26 & 14 \\
Bilateral & 162 & 86 \\
Isolated knee OA & 44 & 23 \\
OA at other sites & & \\
Polyarticular IP OA & 55 & 29 \\
Any IP OA & 112 & 60 \\
1st carpometacarpal OA & 81 & 43 \\
Glenohumeral & 26 & 14 \\
Hip & 25 & 13 \\
Chondrocalcinosis & 62 & 33 \\
\hline
\end{tabular}

$\mathrm{OA}=$ osteoarthritis; IP = interphalangeal. brocker index ${ }^{21}$ and reported exercise tolerance. Knee radiographs were obtained at least annually and change (increase, no change, decrease) in each individual radiographic feature (joint space narrowing, osteophyte, cyst, sclerosis and attrition) was assessed at each knee compartment by comparison of consecutive radiographs (unblinded). An increase was defined as progressive radiographic change for each individual feature. The last available radiograph at completion of the study was assessed in a manner identical to that applied to the study entry film (blinded to the original assessment) and changes in Kellgren grade and scores for each individual feature obtained.

ASSESSMENT OF RADIOGRAPHIC SCORING SYSTEM

Reproducibility for the scoring of individual radiographic features and Kellgren grade (for the same assessors) has already been demonstrated to be good for all features. ${ }^{18}$ Intraobserver variability in scoring for change in each feature on sequential radiographs was assessed for the two observers by the blind rescoring of 50 pairs of consecutive radiographs (100 knees, two sets of radiographs) at completion of the study.

STATISTICAL ANALYSIS

Statistical analysis was by the $\chi^{2}$ and Fisher tests as appropriate. Odds ratios were calculated with respect to the presence or absence of putative associated factors and were calculated with respect to those patients showing no change. Logistic regression was used for continuous variables, and stepwise logistic regression (Egret, Serc, Seattle) for multivariate analysis. Factors included in the models were: patient characteristics (age, sex, body mass index); duration of follow up; nodal change; multiple joint osteoarthritis (involvement of joint sites outside the knee); chondrocalcinosis; and factors found to be associated on univariate analysis. Intraobserver reproducibility was assessed using the K statistic. ${ }^{22}$

\section{Results}

PATIENT CHARACTERISTICS

Prospective clinical and radiographic data (for more than one year) were obtained on 350 osteoarthritic knees (188 patients) from the original 470 knees (254 patients) recruited for cross-sectional assessment. ${ }^{18}$ Follow up was for a mean of 2.26 years (median 2 (range $1-5$ ) years). Few patients (14 knees) underwent knee replacement during the study. Table 1 gives details of patient characteristics.

Obesity, nodal change, polyarticular interphalangeal osteoarthritis, and chondrocalcinosis were common.

At study entry, radiographic osteoarthritis affected $89 \%, 67 \%$, and $14 \%$ of patellofemoral, medial tibiofemoral, and lateral tibiofemoral compartments, respectively. Severe 
Table $2 K$ values for change in individual radiographic features assessed in 50 paired radiographs (100 knees)

\begin{tabular}{llll}
\hline Radiographic feature & $\kappa$ & Agreement & Variance \\
\hline Any & 0.76 & 0.88 & 0.004 \\
Narrowing & 0.74 & 0.88 & 0.005 \\
Osteophyte & 0.68 & 0.89 & 0.008 \\
Cyst & 0.56 & 0.90 & 0.014 \\
Sclerosis & 0.60 & 0.94 & 0.020 \\
Attrition & 0.93 & 0.98 & 0.003 \\
\hline
\end{tabular}

osteoarthritis was present in $27 \%, 23 \%$, and $3 \%$ of these compartments, respectively, and there was fixed flexion deformity in $16(5 \%)$ of knees. These characteristics did not differ significantly in frequency from those seen in the original cross-sectional population.

\section{RADIOGRAPHIC CHANGE}

Reproducibility of the scoring systemsReproducibility for the assessment of change in each feature on sequential radiographs was good for all features assessed, with cystic change being the least reproducible (table 2). Change in scores for each individual feature assessed on the initial and final radiographs showed significant association with change detected by the sequential assessment method. A greater proportion of knees, however, had reduced scores (reduction in total score of $>1$ ) for joint space narrowing, attrition, sclerosis, osteophyte, and cysts $(0 \%, 1 \%, 6 \%, 8 \%$, and $12 \%$, respectively) using the former method.

Kellgren grade-Radiographic progression (defined as increase in Kellgren grade)

Table 3 Odds ratios (OR) and 95\% confidence intervals (CI) for factors associated with change in the individual radiographic features of osteoarthritis $(O A)$ - univariate analysis

\begin{tabular}{|c|c|c|c|}
\hline Feature changed & Associated factor & $O R$ & $95 \% C I$ \\
\hline \multirow[t]{4}{*}{ Kellgren grade } & Nodal change & $1 \cdot 82$ & 1.08 to 3.05 \\
\hline & Polyarticular IP OA & $1 \cdot 89$ & 1.17 to 3.04 \\
\hline & Multiple joint $\mathrm{OA}$ & $2 \cdot 11$ & 1.15 to 3.91 \\
\hline & SF volume* & 1.03 & 1.01 to 1.05 \\
\hline \multirow[t]{6}{*}{ Any } & Effusion & 1.89 & 1.09 to 3.25 \\
\hline & Warmth & $2 \cdot 87$ & 1.66 to 5.00 \\
\hline & SF volume $(>5 \mathrm{ml})$ & $2 \cdot 44$ & 1.46 to 4.09 \\
\hline & CPPD & 1.95 & 1.00 to 4.00 \\
\hline & SF volume* & 1.07 & 1.03 to 1.10 \\
\hline & Severity of knee $\mathrm{OA}^{\star}$ & $1 \cdot 20$ & 1.02 to 1.41 \\
\hline \multirow[t]{3}{*}{ Joint space } & Warmth & $2 \cdot 41$ & 1.52 to 3.82 \\
\hline & SF volume & $2 \cdot 07$ & 1.30 to 3.30 \\
\hline & SF volume ${ }^{\star}$ & 1.04 & 1.02 to 1.07 \\
\hline Osteophyte & SF volume $(>5 \mathrm{ml})$ & 1.82 & 1.09 to 3.04 \\
\hline \multirow[t]{7}{*}{ Cyst } & Nodal & 1.91 & 1.04 to 3.51 \\
\hline & Effusion & $2 \cdot 30$ & $1 \cdot 11$ to $5 \cdot 24$ \\
\hline & Warmth & $3 \cdot 50$ & 1.91 to 6.45 \\
\hline & SF volume $(>5 \mathrm{ml})$ & $2 \cdot 30$ & 1.25 to 4.59 \\
\hline & CPPD & $1 \cdot 01$ & 1.00 to 1.06 \\
\hline & $\mathrm{Age}^{\star}$ & 1.03 & 1.00 to 1.06 \\
\hline & $\mathrm{BMI}^{\star}{ }^{\star}$ & 0.94 & 0.89 to 1.00 \\
\hline \multirow[t]{5}{*}{ Sclerosis } & Effusion & $2 \cdot 35$ & 1.00 to 5.98 \\
\hline & Warmth & $2 \cdot 36$ & 1.21 to 4.64 \\
\hline & SF volume $(>5 \mathrm{ml})$ & $3 \cdot 19$ & $1 \cdot 42$ to $7 \cdot 36$ \\
\hline & $\mathrm{BMI}^{\star}$ & 0.93 & 0.87 to 1.00 \\
\hline & SF volume* & 1.03 & 1.01 to 1.06 \\
\hline \multirow[t]{11}{*}{ Attrition } & Effusion & $2 \cdot 31$ & $1 \cdot 24$ to 4.35 \\
\hline & Warmth & $1 \cdot 83$ & 1.12 to 2.99 \\
\hline & Chondrocalcinosis & $1 \cdot 74$ & 1.05 to 2.88 \\
\hline & CPPD & 3.44 & 1.97 to 6.02 \\
\hline & Nodal & 1.98 & $1 \cdot 15$ to 3.38 \\
\hline & Polyarticular IP OA & 1.95 & $1 \cdot 19$ to $3 \cdot 22$ \\
\hline & SF volume $(>5 \mathrm{ml})$ & $3 \cdot 54$ & 2.00 to 6.33 \\
\hline & $\mathrm{Age}^{\star}$ & 1.04 & 1.02 to 1.07 \\
\hline & SF volume & $1 \cdot 04$ & 1.02 to 1.06 \\
\hline & Severity of knee $\mathrm{OA}^{\star}$ & $1 \cdot 74$ & 1.45 to 2.08 \\
\hline & Pain score ${ }^{\star}$ & $1 \cdot 15$ & 1.01 to 1.31 \\
\hline
\end{tabular}

IP = interphalangeal; $O A=$ osteoarthritis; $S F=$ synovial fluid; $\mathrm{CPPD}=$ calcium pyrophosphate crystals; $\mathrm{BMI}=$ body mass index

Continuous variable.
Table 4 Odds ratios (OR) and 95\% confidence intervals (CI) for factors associated with change in the individual radiographic features of osteoarthritis $(O A)-$ multivariate analysis

\begin{tabular}{llll}
\hline Feature changed & Associated factor & OR & $95 \%$ CI \\
\hline Kellgren grade & Multiple joint OA & 2.39 & 1.16 to 4.93 \\
& SF volume & 1.03 & 1.01 to 1.05 \\
Any & Nodal & 1.80 & 1.02 to 3.17 \\
Woint space & Warmth & 2.22 & 1.19 to 4.14 \\
& BMI $^{\star}$ & 2.08 & 1.25 to 3.46 \\
Osteophyte & BMI $^{\star}$ & 1.07 & 1.02 to 1.14 \\
Cyst & Warmth & 1.06 & 1.00 to 1.12 \\
& Female & 3.03 & 1.63 to 5.64 \\
Sclerosis & SF volume & 2.17 & 1.13 to 4.15 \\
Attrition & Severity of knee OA & 1.03 & 1.01 to 1.05 \\
& CPPD & 1.72 & 1.36 to 2.19 \\
& SF volume & 2.41 & 1.33 to 4.39 \\
& Multiple joint OA & 1.02 & 1.00 to 1.05 \\
& & 2.42 & 1.02 to 5.77 \\
\hline
\end{tabular}

$\mathrm{OA}=$ osteoarthritis; $\mathrm{SF}=$ synovial fluid; $\mathrm{BMI}=$ body mass index; CPPD = calcium pyrophosphate crystals.

$\star$ Continuous variable.

developed in $39 \%$ of knees during follow up. Improvement in Kellgren grade was seen in 19 (5\%) osteoarthritic knees and occurred in the patellofemoral compartment in all except three of these.

Individual radiographic features-Progression in at least one individual osteoarthritis feature was seen on sequential radiographs in a large proportion of knees $(252(72 \%))$. Change occurred in $46 \%$ medial tibiofemoral, $39 \%$ patellofemoral, and $26 \%$ lateral tibiofemoral compartments; in each compartment changes occurred at a median of 1.5 years from study entry. Progression in joint space narrowing occurred in $52 \%$, osteophyte in $32 \%$, attrition in $30 \%$, cysts in $19 \%$, and sclerosis in $14 \%$ of knees.

Reductions in cystic change and sclerosis were seen in a small number of knees; reductions in osteophyte and attrition were not observed on sequential assessment of radiographs. Using this method, increase in joint space was seen only in the tibiofemoral compartments and corresponded with narrowing of the adjacent, worst affected compartment.

Associations-On univariate analysis a variety of factors were found to associate with progression in radiographic features (table 3), but many of these were related and hence lost significance on multivariate analysis. On multivariate analysis (table 4), knees from which synovial fluid was aspirated more commonly showed increase in Kellgren grade, sclerosis and attrition and those with clinical warmth more frequently developed increase in any individual radiographic feature, joint space narrowing, and cystic change. Calcium pyrophosphate crystals and multiple joint osteoarthritis associated with increased attrition.

CHANGE IN SYMPTOMS AND EXERCISE TOLERANCE

Symptoms-Forty eight percent of patients felt their symptoms had deteriorated, but $23 \%$ described improvement. Worsening of symptoms on global assessment (better, same, worse) associated with increase in pain score and disability (Steinbrocker grade), decrease in exercise tolerance, decreased joint space, and increased attrition (table 5). Table 6 shows 
Table 5 Odds ratios (OR) and 95\% confidence intervals (CI) for associated changes in clinical and radiographic features-univariate analysis

\begin{tabular}{|c|c|c|c|}
\hline & Associated factor & $O R$ & $95 \% C I$ \\
\hline \multirow{5}{*}{ Worsening symptoms-global } & Decreased joint space & 1.53 & 1.00 to 2.37 \\
\hline & Increased attrition & 1.95 & $1 \cdot 19$ to $3 \cdot 20$ \\
\hline & Decreased exercise tolerance & $4 \cdot 49$ & $2 \cdot 72$ to $7 \cdot 43$ \\
\hline & Increased disability (Steinbrocker) & 3.59 & 1.61 to 8.20 \\
\hline & Increased pain score & $9 \cdot 32$ & $5 \cdot 31$ to $16 \cdot 5$ \\
\hline \multirow[t]{6}{*}{ Decreased exercise tolerance } & Decreased joint space & $1 \cdot 70$ & 1.07 to 2.73 \\
\hline & Increased sclerosis & $2 \cdot 21$ & $1 \cdot 14$ to $4 \cdot 27$ \\
\hline & Increased attrition & $2 \cdot 13$ & 1.29 to 3.50 \\
\hline & Global worsening of symptoms & $4 \cdot 49$ & $2 \cdot 72$ to $7 \cdot 43$ \\
\hline & Increased pain & $2 \cdot 41$ & 1.48 to 3.94 \\
\hline & Increased disability (Steinbrocker) & $10 \cdot 8$ & 4.35 to 27.9 \\
\hline
\end{tabular}

Table 6 Odds ratios (OR) and 95\% confidence intervals (CI) for factors associated with global change in symptoms and function-univariate analysis

\begin{tabular}{llll}
\hline Feature changed & Associated factor & OR & $95 \%$ CI \\
\hline Worsening symptoms & Effusion & 1.83 & 1.08 to 3.08 \\
-global & Warmth & 2.55 & 1.61 to 4.05 \\
& SF volume $(>5 \mathrm{ml})$ & 1.83 & 1.15 to 2.92 \\
& CPPD & 2.54 & 1.46 to 4.45 \\
& SF volume & 1.03 & 1.01 to 1.05 \\
Increased pain & Severity of knee OA & 1.42 & 1.22 to 1.66 \\
score & CPPD & 1.77 & 1.03 to 3.05 \\
& Chondrocalcinosis & 1.73 & 1.05 to 2.85 \\
Increased disability & Severity of knee OA & 1.18 & 1.01 to 1.37 \\
(Steinbrocker) & Multiple joint OA & 3.39 & 1.00 to 14.2 \\
& Warmth & 2.46 & 1.17 to 5.20 \\
& CPPD & 2.24 & 1.02 to 4.93 \\
& Age & 1.09 & 1.05 to 1.14 \\
Decreased exercise & Severity of knee OA & 1.57 & 1.26 to 1.95 \\
tolerance & Nodal change & 2.25 & 1.33 to 3.80 \\
& Polyarticular IP OA & 2.08 & 1.28 to 3.40 \\
& Effusion & 2.22 & 1.24 to 3.99 \\
& Warmth & 2.56 & 1.58 to 4.12 \\
& CPPD & 2.99 & 1.73 to 5.18 \\
& Bilateral knee OA & 4.62 & 1.28 to 19.7 \\
& Age & 1.04 & 1.02 to 1.07 \\
& SF volume & 1.03 & 1.01 to 1.05 \\
& Severity of knee OA & 1.50 & 1.28 to 1.77 \\
\end{tabular}

$\mathrm{SF}=$ synovial fluid $\mathrm{CPPD}=$ calcium pyrophosphate crystals; $\mathrm{OA}=$ osteoarthritis; IP = interphalangeal.

*Continuous variable.

those characteristics at study entry which associated with deteriorating symptoms on univariate analysis. Significant associations on multivariate analysis were clinical warmth, calcium pyrophosphate crystals, and more severe radiographic change (table 7 ).

Disability-Reported exercise tolerance remained unchanged in the majority $(62 \%)$ and deteriorated in $35 \%$ of patients. Deterioration in reported exercise tolerance associated with increase in attrition, joint space narrowing, and sclerosis (table 5). Several characteristics at study entry associated with deteriorating exercise tolerance on univariate analysis (table 6). Characteristics remaining significantly associated on multivariate analysis were presence of knee warmth, calcium pyrophosphate crystals, and increasing severity of

Table 7 Odds ratios (OR) and 95\% confidence intervals (CI) for factors associated with global change in symptoms and function - multivariate analysis

\begin{tabular}{llll}
\hline Clinical change & Associated factor & OR & $95 \%$ CI \\
\hline Worsening symptoms & Severity of knee $\mathrm{OA}^{\star}$ & 1.29 & 1.07 to 1.57 \\
- global & Warmth & 2.14 & 1.30 to 3.52 \\
& CPPD & 1.89 & 1.06 to 3.38 \\
Increased pain & Severity of knee $\mathrm{OA}^{\star}$ & 1.29 & 1.08 to 1.55 \\
score & CPPD & 1.88 & 1.17 to 3.16 \\
Increased disability & Warmth & 4.25 & 1.66 to 10.9 \\
(Steinbrocker) & Age & 1.07 & 1.02 to 1.13 \\
Decreased exercise & Severity of knee OA* & 1.52 & 1.22 to 1.88 \\
tolerance & Warmth & 2.24 & 1.32 to 1.80 \\
& CPPD & 1.85 & 1.04 to 3.29 \\
\hline
\end{tabular}

$\mathrm{OA}=$ osteoarthritis; $\mathrm{CPPD}=$ calcium pyrophosphate crystals ^Continuous variable. radiographic change (table 7 ). Increase in Kellgren grade, osteophyte, and cysts showed no associations with change in symptoms or disability.

No factors were found to associate with improvement in symptoms or function. On multivariate analysis, symptom improvement was associated negatively with warmth, calcium pyrophosphate crystals, and more severe radiographic change (odds ratios 0.53 , 0.41 , and $0.75 ; 95 \%$ CI 0.29 to $0.95,0.18$ to 0.90 , and 0.60 to 0.94 , respectively). Increasing age showed inverse association with improvement in function (odds ratios 0.88 , $95 \%$ CI 0.82 to 0.95 ).

\section{Discussion}

This large prospective study has identified several factors that are predictors of radiographic and clinical change in knee osteoarthritis of patients referred to hospital.

Approximately $50 \%$ of the patients described worsening of their symptoms with time though, as previously emphasised, ${ }^{6} 152324$ a significant proportion described improvement. Assessment of treatment was not the purpose of this study. Multiple interventions (for example non-steroidal anti-inflammatory drugs, intra-articular injections, physiotherapy, advice on exercise and diet) had been used before or during the study, making correction for and analysis of individual interventions impossible. Many factors influence symptom reporting, and correlation between pain and structural abnormality in cross-sectional surveys is often poor. ${ }^{25}{ }^{26}$ In this longitudinal study, however, deterioration in symptoms was associated with change in radiographic features and in particular with increasing bone attrition. Change in function, as measured by exercise tolerance, also showed a strong association with increasing attrition. Interestingly, increasing osteophytosis failed to associate with worsening symptoms or function. Although osteophyte has been incriminated as a cause of symptoms, this present finding supports the view that new bone formation may be a 'beneficial' feature of the osteoarthritic process. Conversely, the association between clinical deterioration (symptoms, function) and progression of bone attrition and joint space narrowing suggest that both cartilage and bone loss are detrimental, possibly reflecting failure of joint homeostasis.

The association between knee effusion, particularly warm effusions, and increase in each of the five radiographic features assessed is of interest. These associations could reflect a primary pathological role for synovial fluid with deleterious effects on bone and cartilage resulting from inflammatory factors in synovial fluid or increased intra-articular pressure. Alternatively, the effusions could be secondary and reflect a more aggressive osteoarthritic process with 'debris synovitis'. The possibility that the use of intra-articular injections of steroid in these knees is related to the radiographic progression requires further specific investigation. 
This is the first prospective study of knee osteoarthritis to examine synovial fluid systematically for the presence of calcium pyrophosphate crystals. Cross-sectional studies have previously linked calcium pyrophosphate crystals with cystic change. ${ }^{911} 12$ This study has now associated these crystals with progressive cystic change, progressive bone attrition, and poor symptomatic and functional outcome. Crystal deposition is not an 'all or none' phenomenon ${ }^{27}$ and lack of detection does not exclude their presence in the joint. Quantification of crystals is problematic. ${ }^{112728}$ However, ready detection of crystals in a fluid by means of compensated polarised microscopy implies a greater crystal load than in fluids with no crystals identified by this means. As with effusion, the presence of plentiful calcium pyrophosphate crystals could result in inflammatory and mechanical insult, or be a secondary consequence of extensive and severe osteoarthritis reflecting a 'field change' in the cartilage matrix. ${ }^{28}$

There are a number of caveats to this study. It is unclear how far results from patients with symptoms severe enough to warrant hospital referral may be extrapolated to community based subjects with knee osteoarthritis. This study has demonstrated a greater degree of radiographic progression than previously reported in community based ${ }^{4}$ and hospital based studies. ${ }^{6}{ }^{15-17}$ This may in part reflect the subjects studied; ours is the largest hospital based study to be reported and the only one to include sequentially referred patients. The studies by Massardo et $a l^{15}$ and Spector et al ${ }^{16}$ were reviews of cohorts previously enrolled for the assessment of short term symptomatic outcome with drug intervention-inclusion criteria that may have resulted in exclusion of more severely affected individuals. Patients in this study were also older than those included in previous studies ${ }^{4}$ and this may be a factor in the lack of obvious association between obesity and progression in this study.

The method of radiographic assessment used has a major effect on the results of such a study. Unfortunately, there is no accepted standard for assessment of structural change in osteoarthritis. We therefore used both a commonly used global scoring system and a system that assessed individual radiographic features. As previously reported, ${ }^{29}$ the Kellgren and Lawrence global system suffers from lack of sensitivity to change and inability to record further change in knees with the most severe grade; in addition, it assumes that different features progress together. In this study we found associations between clinical outcome (symptoms, disability) and change in some, rather than all, of the radiographic features of osteoarthritis. Although the global system is robust, grading of individual features may be more sensitive to change and more useful in interpreting structural change in osteoarthritis.

The radiographic views obtained are also important. Only two previous hospital based studies ${ }^{15} 17$ are comparable to the current study on the basis of the radiographs obtained (weight bearing anteroposterior and lateral views); the severity of radiographic osteoarthritis at study entry was not comparable in these studies, however, the subjects in the current study having more severe change ${ }^{17}$ and more associated disability. ${ }^{15}$ The patellofemoral joint was found to be the most commonly affected compartment in this study. Patellofemoral assessments have not been undertaken in the majority of previous studies and this also may contribute in part to any differences in results.

Examination of successive radiographs to detect change is subject to time bias and may have contributed to the greater number of patients showing progression. Random noise is unlikely to have a major effect because a large number of patients were studied and there was a predominance of change in one direction only. Similarly, observer variability, whilst an important consideration, was generally good for all features assessed.

Multiple statistical tests were performed and this may have produced spurious significant associations. This may have been partially allowed for by the use of multivariate analysis, but the results obtained from this study need to be replicated in other similar populations.

This large study has demonstrated change in radiographic features in the majority of patients referred to hospital because of osteoarthritis of the knee. Deterioration in symptoms and function occurred in a smaller proportion of patients and was associated with change in specific radiographic features. The presence of knee effusions, clinical warmth, calcium pyrophosphate crystals, and increasing severity of specific radiographic features at the time of hospital referral were associated with poor symptomatic, radiographic, and functional outcome. Such factors should be taken into account in future long term studies of knee osteoarthritis, particularly those involving intervention.

JL is a Roussel (U.K.) Osteoarthritis Research Fellow. We are grateful to Trent Regional Health Authority and the Arthritis and Rheumatism Council for financial support.

1 Kellgren J, Moire R. Generalised osteoarthritis and Heberden nodes. BMF 1952; 1: 180-7.

2 Ahlbach S. Osteoarthritis of the knee-A radiographic investigation. Acta Radiologica 1968; 277: 6-71.

3 Kannus B, Jarvinen M, Salminon E, et al. Occurrence of symptomatic knee osteoarthrosis in rural Finland: a prospective follow-up study. Ann Rheum Dis 1987; 46 804-8.

4 Schouten J S A G, Van den Ouweland F A, Valkenburg H A A 12 year follow-up study in the general population on prognostic factors of cartilage loss in osteoarthritis of the knee. Ann Rheum Dis 1992; 51: 932-7.

5 Cushnaghan J, Dieppe P. Study of 500 patients with limb joint osteoarthritis 1 . Analysis by age, sex and distribution of symptomatic joint sites. Ann Rheum Dis 1991; 50: 8-13.

6 Hernborg J S, Nilsson B E. The natural course of untreated osteoarthritis of the knee. Clin Orthop Rel Res 1977; 123: $130-7$.

7 Thomas R H, Resnick D, Alazraki N P, Davies D Greenfield R. Compartmental evaluation of osteoarthritis of the knee. A comparative study of available diagnostic modalities. Radiology 1975; 16: 585-94.

8 Peyron J G. Epidemiological and aetiologic approach of osteoarthritis. Semin Arthritis Rheum 1979; 8: 288-306.

9 Resnick D, Niwayama G, Goergen T G, et al. Clinical, radiographic and pathologic abnormalities in calcium pyrophosphate dihydrate deposition disease (CPPD): pseudogout. Radiology 1977; 122: 1-15.

10 Gorden T P, McCredie M, Smith M, Brooks P M Ebert B. Articular chondrocalcinosis in a hospital population-an Australian experience. Aust $N Z \mathcal{F} \mathrm{Med}$ 1984; 14: 655-9.

11 Doherty $M$, Dieppe P A. Clinical aspects of calcium pyrophosphate dihydrate crystal deposition. Rheum Dis Clin N Am 1988; 14: 395-414. 
12 Halverson P, McCarty N J. Patterns of radiographic abnormalities associated with basic calcium phosphate and calcium pyrophosphate dihydrate deposition in the and calcium pyrophosphate dihydrate
knee. Ann Rheum Dis 1986; 45: 603-5.

13 Felson D. The epidemiology of knee osteoarthritis: results from the Framingham study. Semin Arthritis Rheum 1990; 20: $42-50$

14 Davis M A. The association of knee injury and obesity with unilateral and bilateral osteoarthritis of the knee. $A m \mathcal{F}$ Epidemiol 1989; 130: 278-88.

15 Massardo L, Watt I, Cushnaghan J, Dieppe P. Osteoarthritis of the knee joint: an eight year prospective study. Ann Rheum Dis 1989; 48: 893-7.

16 Spector T D, Dacre J E, Harris P A, Huskisson E C. Radiological progression of osteoarthritis: an 11 year follow up study of the knee. Ann Rheum Dis 1992; 51: 1107-10.

17 Dieppe P, Cushnaghan J, Young P, Kirwan J. Prediction of the progression of joint space narrowing in osteoarthritis of the knee by bone scintigraphy. Ann Rheum Dis 1993 52: $557-63$.

18 Ledingham J M, Regan $M$, Jones A, Doherty $M$ Radiographic patterns and associations of knee osteoarthritis in a hospital referred population. Ann Rheum Dis 1993; 52: 520-6.

19 Kellgren J, Lawrence J S. Radiological assessment of osteoarthritis. Ann Rheum Dis 1957; 16: 494-502.

20 Solomon L. Patterns of osteoarthritis of the hip. F Bone foint Surg Br 1976; 58: 176-83.
21 Steinbrocker O, Traeger C H, Battman R C. Therapeutic criteria for rheumatoid arthritis. fAMA 1949; 140: 659-62. Cohen J. A coefficient of agreement for nominal scales. $E d u c$ Psychol Meas 1960; 20: 37-46.

23 Berkhout B MacFarlane J D, Cats A. Symptomatic osteoarthritis of the knee: A follow up study. $\mathrm{Br} \mathcal{F}$ Rheumatol 1985; 24: 40-5.

24 Danielsson L, Hernborg J. Clinical and roentgenologic study of knee joints with osteophytes. Clin Orthop Res 1970; 69: 302-12.

25 Dieppe P A. Why is there such poor correlation between radiographic joint damage and both symptoms and functional impairment in osteoarthritis? $\mathrm{Br} \mathcal{F}$ Rheumatol 1989; 28: 242 .

26 Lawrence J S, Bremner J M, Bier F. Osteoarthrosis: prevalence in the population and relationship between symptoms and X-ray changes. Ann Rheum Dis 1966; 25: $1-24$.

27 Hamilton E Pattrick M, Hornby J, Derrick G, Doherty $M$. Synovial fuid calcium pyrophosphate dihydrate crystals Synovial fluid calcium pyrophosphate dihydrate crystals fRheumatol 1990; 29: 101-4.

28 Pattrick M, Hamilton E, Wilson R, Austin S, Doherty M. Association of radiographic changes in osteoarthritis and synovial fluid particles in 300 knees. Ann Rheum Dis 1993; 52: 97-103.

29 Ledingham J M, Dawson S, Preston B, Doherty M. Radiographic progression of hospital referred hip osteoarthritis. Ann Rheum Dis 1993; 52: 263-7.

\section{(ำ

$\frac{1}{3}$ 